\title{
ZEEMAN SPLITTING OF EXCITED BORON STATES IN p-Ge
}

\author{
G. Jungwirt and W. Prettl \\ Institut für Angewandte Physik, Universität Regensburg \\ D-8400 Regensburg, West Germany.
}

Received June 1, 1989

The photothermal spectrum of shallow acceptors in p-Ge has been investigated at various magnetic field strengths up to $5.6 \mathrm{~T}$ at a temperature of $7.5 \mathrm{~K}$ by FIR-Fourier-spectroscopy. From the observed Zeeman splittings of the excited states of the boron acceptor the coefficients of the linear and quadratic field dependence have been evaluated and $g$-factors of the $D$-, $C$ - and the $G$-transitions have been determined based on a standard group theoretical approach.

Key words : boron doped p-Ge, shallow acceptors, Zeeman-splitting

\section{Introduction}

The optical excitation spectra of hydrogen-like shallow impurities in semiconductors may be determined by photo-thermal ionization spectroscopy (PTIS) at low temperatures. Charge carriers optically excited to bound impurity states are transferred to the corresponding conduction or valence band by absorption of residual thermal phonons yielding a sensitive photoconducting response even in materials with very low impurity concentration [1]. Numerous investigations of various semiconductor materials have been performed giving detailed knowledge of the electronic structure of shallow impurities in particular of the effect of an external magnetic field on the energy levels [2]. Soepangkat and Fisher [3] analyzed the Zeeman splitting of acceptor absorption lines in $G e$, while Broeckx et al. [4] observed the Zeeman effect of the $D$ - and $C$-transitions of aluminium in $G e$ applying PTIS in the magnetic field range of $0.3-2.5 \mathrm{~T}$ and Jongbloets et al. [5] investigated the boron acceptor with magnetic fields up to $2.0 \mathrm{~T}$. As the $g$-factor of shallow acceptor ground states was shown to be vanishingly small $[5,6]$, the $g$-factors of excited states can be determined from the observed line 
splittings. In the present work the photothermal ionisation spectra of predominantly boron doped $p-G e$ were investigated up to $5.6 \mathrm{~T}$ extending earlier measurements. The $g$-factors of the $D$ - and $C$ lines could be determined with better reliability than previously and additionally the magnetic field splitting of the $G$-line has been observed.

\section{Technique}

The investigated sample was prepared from a $\mathrm{p}-\mathrm{Ge}$ single crystal with faces normal to the [111] crystallographic axis. The effective acceptor concentration and the compensation ratio were 2.5 . $10^{12} \mathrm{~cm}^{-3}$ and $1 \%$, respectively. The sample contained the shallow acceptors boron and aluminium with a density ratio $n_{B} / n_{A l}=10$. Ohmic contacts were made on the sample by alloying $\mathrm{Al}$ on opposite edges. The sample was mounted at the end of a metallic light pipe fitted in a variable temperature cryostat in the center of a superconducting magnet. The photothermal conductivity spectrum of the sample was measured in Faraday configuration with the magnetic field normal to the [111] faces of the crystal, using a commercial far-infrared Fourier-spectrometer in the frequency range between 45 and $200 \mathrm{~cm}^{-1}$. A standard load resistor circuit was used and the bias roltage was kept well below the critical voltage of impurity breakdown to avoid impact ionisation instabilities in the photoconductive response [7].

\section{Experimental results}

The measurements were carried out up to $B=5.6 \mathrm{~T}$. In Fig. 1 photoconductivity spectra obtained at a temperature of $7.5 \mathrm{~K}$ are shown for four different magnetic field strengths. The chemical species of the involved acceptors can be identified from the well known. spectral pattern of the zero magnetic field lines [8]. Different shallow acceptors yield the same spectral structure shifted relatively to one another by central cell effects of the ground state [2]. The labelling of the lines in Fig. 1 is according to [9]. The local symmetry of an acceptor on a $G e$ lattice site corresponds to the point group $T_{d}$. In the effective mass approximation however, the small terms in the impurity potential being non-symmetric against inversion are neglected and the acceptor states are characterised by double group representation of the octahedral groups $O_{h}$, having definite parity [10]. Applying this classification scheme, all observed transitions originate from the $\Gamma_{8}^{+}$acceptor ground state. The $G-, D$ - and $B$ lines were attributed to $\Gamma_{8}^{-}$end states, whereas the $C$-line is due to two excited states, $\Gamma_{8}^{-}$and $\Gamma_{\overline{7}}^{-}$, being energetically very close [10]. The $A$ - and $I$-lines are actually groups of 4 and 8 lines at $B=0 \mathrm{~T}$, respectively. They will not be discussed further because due to the 




Fig. 1 Photoconductivity spectra of boron-doped p-Ge for various magnetic field strengths parallel to [111]. The labelling of the lines is according to [9], primed labels indicate aluminium excitations. The lines assigned by $X$ at high magnetic fields are likely to belong to the boron $B$-multiplet. The threshold of direct photoionisation for boron is marked by $E_{I}$. 
large number of overlapping components the splittings in a magnetic field cannot be uniquely identified. The binding energy of acceptors cannot be experimentally determined from the photoconductivity spectra. The threshold of direct photoionisation out of the ground state for Boron is expected to be at $87.3 \mathrm{~cm}^{-1}[8]$.

The measurements plotted in Fig. 1 demonstrate the shifting and splitting of optical transitions below the photoionisation continuum. The spectral position of uniquely identified lines as functions of the magnetic field strength is displayed in Fig. 2. At low fields the

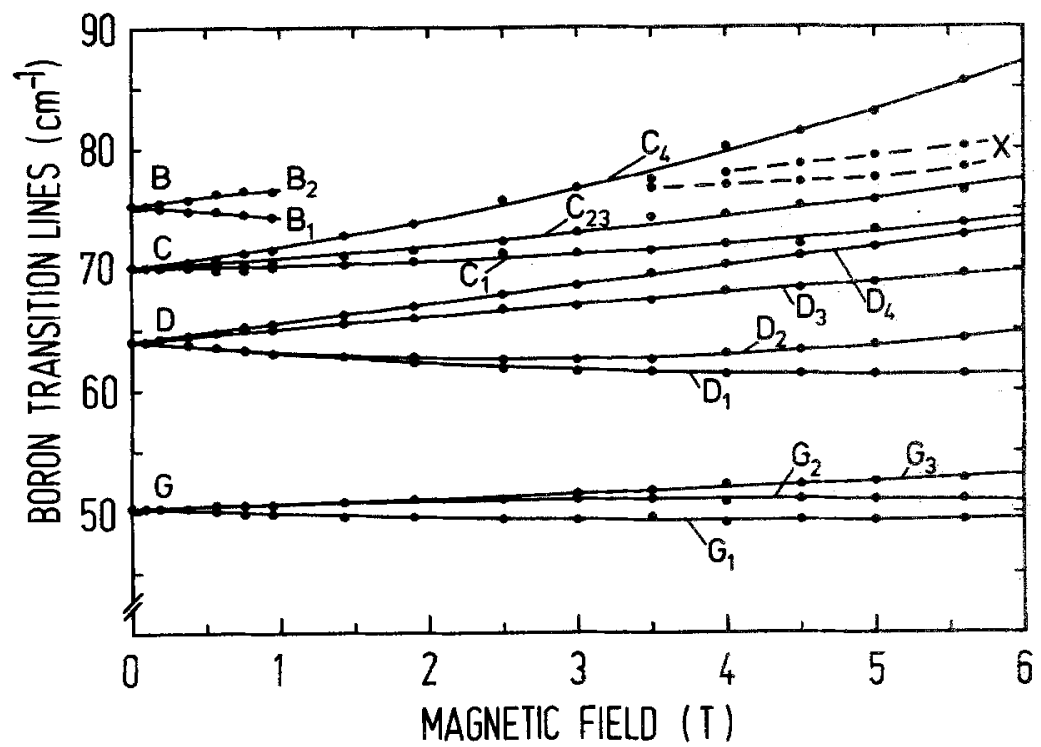

Fig. 2 Zeeman splitting of the $G$-, $D$ - and $C$-lines for the boron acceptor in $G e$ with the magnetic field parallel to [111]. The splitting of the $B$-line could be followed only up to $1.0 \mathrm{~T}$, but it is supposed that the lines assigned with $X$ are the continuation of the $B$ multiplet.

$D$-line splits into two components being symmetrical with respect to its zero-field position, while the $C$ - line splits into a triplet. The $B$ and $G$-transitions also split into two lines. At the highest fields all four components of the $D$-line are clearly identifiable and the $G$-line shows three components. The energetically highest component of the $C$-transitions, $C_{4}$, shows an extremely strong shift to higher energy, crossing many lines resulting from the $B-, A$ - and $I$-multiplets, on the other hand the signal of the energetically lowest line of $C$ almost vanishes at higher fields, whereas the nearby $D_{4}$ sublevel grows in 
strength. At magnetic fields higher than $3.0 \mathrm{~T}$, the $C_{2 s}$ sublevel seems to split again and in the right shoulder of this peak a new line appears. Later on, at 5.0 T an additional structure shows up at the left shoulder. According to a detailed analysis of the $C$-line in germanium by Broeckx et al. [4] no splitting of the $C_{2 s}$ peak should occur. However, after calculations of Lipari and Baldereschi [10] there exists a $\Gamma_{\overline{7}}^{-}$state energetically almost coinciding with the $\Gamma_{8}^{-}$end state of the $C$-line. This $\Gamma_{7}^{-}$state has been reported by Gershenzon [11], but it could never be detected by PTIS-measurements, although the resolution in these experiments has been sufficiently large (see references in [12]). This $\Gamma_{7}^{-}$-state might appear at high magnetic fields or perhaps there might be some admixture with sublevels from this state. For high magnetic fields (for example $5.6 \mathrm{~T}$ ) two strong peaks can be detected between the $C_{23}$ and $C_{4}$ line (broken line in Fig. 2). It cannot be decided, whether these lines belong to the $B$ multiplet or are eventually $A$-lines. The magnetic field dependence of the $B$-line is difficult to follow because of the strong $C$-line, which crosses the $B$-line and because of the many $A$-lines occuring in this spectral range.

In Fig. 2 the results of the measurements with $B$ being parallel to the [111] crystallographic orientation are displayed. The experimental peak positions of the transitions are plotted as a function of the magnetic field. To fit the experimental data, shown by dots in Fig. 2, we used a polynomial of second order in the magnetic field st rength $\Delta E=E(B)-E(0)=a B+b B^{2}$ taking into account the linear and quadratic Zeeman effects.

The parameters $a$ and $b$ for the various lines are summarized in Tab. 1. The experimental results of Broeckx et al. [4] for the $C$ and $D$-transitions of aluminium are also presented in this table.

The results of both investigations are significantly different which is not expected by this amount even for chemically different acceptors. The parameters of [4] fit our measurements quite well for magnetic fields up to about $2.5 \mathrm{~T}$ however not for the whole field range of the present measurements. This difference in fit parameters is especially important for the interpretation of the $D$ line. Broeckx et al. assumed a crossing of the two lowest $D$ levels, with the consequence that they had to attribute the magnetic quantum number $m_{j}=3 / 2$ for calculating the $g$-factors of the $D$-line to the upper level. Our analysis does not yield a crossing of these two lines, and therefore we have to take the lower level to determine the $g$-factor $g_{3 / 2}^{\prime}$. The $B$-line splits into two components, but, as they are observable only up to $1.0 \mathrm{~T}$, a reasonable evaluation of the measurements is not possible. 
Table 1: Linear and quadratic Zeeman parameters in the $\$$ || [111] orientation for the $B-, C_{-}, D_{-}$, and the $G$-lines of the boron acceptor in $G e$ obtained from measurements up to 5.6 T. Previous results of Broeckx et al. [4] are also given for the purpose of comparison.

\begin{tabular}{|c|c|c|c|c|c|}
\hline \multicolumn{3}{|c|}{ present investigation Ge(B) } & \multicolumn{3}{|c|}{ Broeckx et al. Ge(Al) } \\
line & $\mathrm{a}(\mathrm{meV} / \mathrm{T})$ & $\mathrm{b}\left(\mathrm{meV} / \mathrm{T}^{2}\right)$ & line & $\mathrm{a}(\mathrm{meV} / \mathrm{T})$ & $\mathrm{b}\left(\mathrm{meV} / \mathrm{T}^{2}\right)$ \\
\hline$B_{2}^{*}$ & 0.2068 & -0.0362 & & - & - \\
$B_{1}^{*}$ & -0.1277 & 0.0038 & & - & - \\
$C_{4}$ & 0.1891 & 0.0269 & $C_{1}^{\prime}$ & 0.1571 & 0.0424 \\
$C_{23}$ & 0.0868 & 0.0109 & $C_{32}^{\prime}$ & 0.0331 & 0.0301 \\
$C_{1}$ & 0.0055 & 0.0135 & $C_{4}^{\prime}$ & -0.0645 & 0.0481 \\
$D_{4}$ & 0.1919 & 0.0007 & $D_{1}^{\prime}$ & 0.1671 & 0.0072 \\
$D_{3}$ & 0.1318 & -0.0018 & $D_{2}^{\prime}$ & 0.1264 & -0.0057 \\
$D_{2}$ & -0.1351 & 0.0253 & $D_{4}^{\prime}$ & -0.1821 & 0.0432 \\
$D_{1}$ & -0.1371 & 0.0138 & $D_{3}^{\prime}$ & -0.1528 & 0.0168 \\
$G_{3}$ & 0.0409 & 0.0028 & & - & - \\
$G_{2}$ & 0.0471 & -0.0059 & & - & - \\
$G_{1}$ & -0.0622 & 0.0075 & & - & - \\
\hline
\end{tabular}

${ }^{*}$ the fit for the $B$-sublevels was carried out only up to $B=1.0 \mathrm{~T}$

\section{Discussion}

Bhattacharjee and Rodriguez [13] described the splitting of the $\Gamma_{6}^{-}, \Gamma_{7}^{-}$and $\Gamma_{8}^{-}$acceptor levels (again $O_{h}$ notation is used) in homogeneous magnetic fields parallel to the [111], [100] and [110] crystallographic directions based on a group-theoretical treatment. Each symmetry species was separately analyzed by firstorder degenerate perturbation theory. Experimentally we find that magnetically split acceptor states belonging to the same irreducible representation do not come so close that interaction must be taken into account. Thus this model may be adopted to evaluate the present measurements. The general expression for the Zeeman Hamiltonian projected onto the four-dimensional subspace formed by the basis functions of a $\Gamma_{8}^{-}$level is [13]:

$$
\begin{aligned}
H_{Z}^{(8)}= & \mu_{B} g_{1}^{\prime} B \cdot J+\mu_{B} g_{2}^{\prime}\left(B_{x} J_{z}^{3}+B_{y} J_{y}^{s}+B_{z} J_{z}^{s}\right)+ \\
& q_{1} B^{2} 1_{4}+q_{2}(B \cdot J)^{2}+q_{s}\left(B_{x}^{2} J_{z}^{2}+B_{y}^{2} J_{y}^{2}+B_{z}^{2} J_{z}^{2}\right)
\end{aligned}
$$

where $J_{i}$ with $i=x, y, z$ are matrices belonging to the angular momentum $J=3 / 2,1_{4}$ is the 4 -dimensional unit matrix and $\mu_{B}$ 
the Bohr magneton.

Tokumoto et al. [5] reported a small splitting of the ground state of shallow acceptors in $G e$. In recent experiments however Freeth et al. [6] found that the $g$-factor of the ground state is zero. Broeckx et al. [4] pointed out that the ground state splitting even taking the results of Ref. [5] is so small that the measured line splitting reflects only the effect of the magnetic field on the excited states. With our analysis of the $g$-factors we will follow the same procedure. The principal $g$ - factors of a $\Gamma_{\overline{8}}^{-}$level expressed in terms of $g_{1}^{\prime}$ and the parameter $\mathrm{r}=g_{2}^{\prime} / 4 g_{1}^{\prime}$ (which measures the cubic anisotropy) are given by [14]:

$$
\begin{aligned}
& g_{3 / 2}^{\prime}=1 / 3 g_{1}^{\prime} r\left[(3 / r+23)^{2}+32\right]^{1 / 2} \\
& g_{1 / 2}^{\prime}=g^{\prime}(1+13 r)
\end{aligned}
$$

yielding the linear energy shifts :

$$
\begin{aligned}
& \Delta E_{ \pm \mathbf{3} / 2}^{(8)}= \pm 3 / 2 g_{\mathbf{3} / 2}^{\prime} \mu_{B} B \\
& \Delta E_{ \pm 1 / 2}^{(8)}= \pm 1 / 2 g_{1 / 2}^{\prime} \mu_{B} B
\end{aligned}
$$

The final state of the $D$-transition can be assigned by $2 \Gamma_{8}^{-}$according to Lipari and Baldereschi [10]. Broeckx et al. identified the $D$ multiplet with $B$ parallel to [111] as the transitions from the unsplit $\Gamma_{8}^{+}$ground state to final states $\Gamma_{8}^{-}\left(m_{j}\right)$ as

$$
\begin{aligned}
& D_{1}: \Gamma_{8}^{+} \rightarrow \Gamma_{8}^{-}(+3 / 2) \\
& D_{2}: \Gamma_{8}^{+} \rightarrow \Gamma_{8}^{-}(+1 / 2) \\
& D_{3}: \Gamma_{8}^{+} \rightarrow \Gamma_{8}^{-}(-1 / 2) \\
& D_{4}: \Gamma_{8}^{+} \rightarrow \Gamma_{8}^{-}(-3 / 2)
\end{aligned}
$$

(corresponding to $g_{1}^{\prime}>0$; for $g_{1}^{\prime}<0$ all the signs should be reversed). Following the argument of Ref. [4] we find for the $g_{3 / 2}^{\prime}$ and $g_{1 / 2}^{\prime}$ from the measured linear Zeeman parameters for different sublevels:

$$
\begin{aligned}
& g_{3 / 2}^{\prime}(111)=\left(a\left(D_{1}\right)-a\left(D_{4}\right)\right) / 3 \mu_{B} \\
& g_{1 / 2}^{\prime}(111)=\left(a\left(D_{2}\right)-a\left(D_{3}\right)\right) / \mu_{B}
\end{aligned}
$$

As the lines $D_{1}$ and $D_{2}$ do not cross we attribute the lowest $D$ level, $D_{1}$, as the transition $\Gamma_{8}^{-}(3 / 2)$. These phenomenological $g$ values permits the calculation of $g_{1}^{\prime}$ and $r$. The results are listed in Tab. 2. For comparison the calculated values of Broeckx et al. are also given. 
Table 2: Calculated $g$-factors of the linear Zeeman effect for the final states of the $D$-, $C$ - and $G$-transition with $\mathbf{B} \|[111]$ for the boron acceptor in Ge. Also given are the results of Broeckx et al. [4] for Aluminium.

\begin{tabular}{|c|c|c|c|c|}
\hline & $g_{3 / 2}^{\prime}$ & $g_{1 / 2}^{\prime}$ & $g_{1}^{\prime}$ & I \\
\hline$D:\left\{\begin{array}{l}G e(B): \\
G e(A l):\end{array}\right.$ & $\begin{array}{l} \pm 1.90 \\
\pm 2.01\end{array}$ & $\begin{array}{l} \pm 4.61 \\
\pm 4.82\end{array}$ & $\begin{array}{l} \pm 7.9 \\
\pm 8.4\end{array}$ & $\begin{array}{l}-0.122 \\
-0.121\end{array}$ \\
\hline$C:\left\{\begin{array}{l}G e(B): \\
G e(A l):\end{array}\right.$ & $\begin{array}{l} \pm 1.06 \\
\pm 1.27\end{array}$ & $\begin{array}{l}0 \\
0\end{array}$ & $\begin{array}{l} \pm 2.4 \\
\pm 2.9\end{array}$ & $\begin{array}{l}-1 / 13 \\
-1 / 13\end{array}$ \\
\hline$G: \operatorname{Ge}(B):$ & \pm 0.59 & 0 & \pm 1.4 & $-1 / 13$ \\
\hline
\end{tabular}

The value of the anisotropy factor $r$ is in good agreement with Broeckx et al. [4], being close to the theoretical value $r=-5 / 41=$ -0.1219 . For the $C$-transition we assume a $\Gamma_{8}^{-}$final state, neglecting the additional peaks seen at high magnetic fields. For the present orientation of $\mathrm{B}$ the observed splitting of the $C$ - line into a triplet by itself determines uniquely the anisotropy factor to be $r=-1 / 13$. Therefore the sublevels of the $C$-triplet may be assigned by:

$$
\begin{aligned}
& C_{1}: \Gamma_{8}^{+} \rightarrow \Gamma_{8}^{-}(+3 / 2) \\
& C_{23}: \Gamma_{8}^{+} \rightarrow \Gamma_{8}^{-}( \pm 1 / 2) \\
& C_{4}: \Gamma_{8}^{+} \rightarrow \Gamma_{8}^{-}(-3 / 2)
\end{aligned}
$$

(corresponding to $g_{1}^{\prime}>0$ ).

From the measured linear Zeeman parameters again we obtain the phenomenological $g$-factors, which are given in Tab. 2 . The anisotropy factor $r$ is in agreement with Broeckx et al. [4], whereas $g_{1}^{\prime}$ for the $D$ - and $C$-transitions is somewhat smaller. The final state of the $G$-transition can be assigned $1 \Gamma_{8}^{-}$according to Lipari and Baldereschi [10]. There exist only absorption measurements of Soepangkat and Fisher [3] for this line with the magnetic field parallel to [111], but they have seen only a splitting into a doublet. As shown in Fig. 2 the $G$-line splits into a triplet similar to the $C$-line. Therefore we use here the same procedure assigning the sublevels by the transitions:

$$
\begin{aligned}
& G_{1}: \Gamma_{8}^{+} \rightarrow \Gamma_{8}^{-}(+3 / 2) \\
& G_{2}: \Gamma_{8}^{+} \rightarrow \Gamma_{8}^{-}( \pm 1 / 2) \\
& G_{3}: \Gamma_{8}^{+} \rightarrow \Gamma_{8}^{-}(-3 / 2)
\end{aligned}
$$

(corresponding to $g_{1}^{\prime}>0$ ).

Again the triplet character uniquely determines the anisotropy parameter to be $r=-1 / 13$. The phenomenological $g$-factors are 
listed in Table 2, together with the values of $r$ and $g_{1}^{\prime}$. The value $r=-1 / 13$ corresponds to a splitting of the $G$-line for the magnetic field parallel to [100] into a doublet and for $B$ parallel to [110] into a quartet. Soepangkat et al. have seen a splitting into a doublet for the [100] direction of the magnetic field, but they used magnetic fields up to $2.1 \mathrm{~T}$ only, so the splitting may be resolvable at higher magnetic fields.

In summary the Zeeman splitting of optical transitions between the ground state and excited states of boron acceptors in high purity $G e$ has been investigated in a more extended magnetic field range than before. Assuming the ground state $g$-factor to be unresolvable small, the magnetic field dependence of the excited states can be described by a linear and a quadratic term in the field strength. Based on the theory of Bhattacharjee and Rodriguez [13] reliable $g$-factors for the $2 \Gamma_{8}^{-}$and $3 \Gamma_{8}^{-}$states, corresponding to the line $D$ and $C$ respectively, were determined. Finally a triplet structure of the $G$-line could be uniquely established yielding the corresponding $g$-factors.

\section{Acknowledgement}

We thank E.E.Haller for provision of the germanium crystal. Financial support by the Deutsche Forschungsgemeinschaft is gratefully acknowledged.

\section{References}

[1] Lifshits T.M. and Nad F.Ya., Sov. Phys.-Dokl. 10532 , (1965).

[2] Bassani F., Iadonisi G. and Preciosi B., Rep.Prog.Phys 37, 1099 (1974).

[ 3] Soepangkat H.P.and Fisher P., Phys.Rev. B 8, 870 (1973).

[4] Broeckx J., Clauws P., Van den Steen K. and Vernik J., J.Phys. C: Solid St. Phys. 12, 4061 (1979).

[5] Tokumoto H. and Ishiguro T., Phys.Rev. B 15, 2099 (1977).

[6] Freeth C.A., Fisher P. and Simmonds P.E., Solid St. Commun. 60, 175 (1986).

[7] Schöll E., Heisel W. and Prettl P., Z. Phys. B 47, 285 (1982).

[8] E.E.Haller and Hansen W.L., Solid St. Commun. 15, 687 (1974).

[9] Jones R.L. and Fisher P., J.Phys.Chem.Solids 26, 1125 (1965).

[10] Lipari N.O. and Baldereschi A., Solid St. Commun. 25, 665 (1978).

[11] Gershenzon E.M. Gol'tsman G.N. and Kagane M.L., Sov. Phys.-JETP 45, 769 (1977). 
[12] Kogan S.M. and Lifshits T.M., Phys.Stat.Solidi.(a) 39, 11 (1977).

[13] Bhattacharjee A.K. and Rodriguez S., Phys.Rev. B 6, 3836 (1972).

[14] Merlet F., Pajot B., Arcas P. and Jean-Louis A.M., Phys.Rev.B 12,3297 (1975). 